\title{
Strafrechtlicher Schutz des Wettbewerbs: Ein Nachweis normativer Fehlregulierung des Marktes*
}

Von Korruption im Wirtschaftsverkehr liest und hört man immer wieder - Meldungen in den Medien offenbaren und erwecken den Eindruck, Strafverfolgungsorgane deckten vermehrt Schmiergeldsachverhalte auf. Man liest von Schmiergeldzahlungen bei Vergabe von Bauaufträgen, von Bestechungspraktiken in Großkonzernen oder im kommunalen Bereich. Preisabsprachen in verschiedenen Wirtschaftszweigen, vor allem in der Bauwirtschaft, beschäftigen Strafverfolgungsbehörden und die Öffentlichkeit schon seit Jahrzehnten. ${ }^{1}$ Das öffentliche Interesse von Medien, Politik und Bevölkerung an der Korruption, die den wirtschaftlichen Wettbewerb angeblich zu unterwandern droht, ist groß. ${ }^{2}$ Daher haben insbesondere politische Vorschläge, wirtschaftliche Problemlagen mit den Mitteln des Strafrechts zu lösen, in den letzten Jahrzehnten stetig zugenommen. ${ }^{3}$ Bereits am 20. August 1997 trat das Gesetz zur „Bekämpfung““4 der Korruption in Kraft, das unter anderem die Straftatbestände zum Schutz des Wettbewerbs als 26. Abschnitt in das Strafgesetzbuch (StGB) eingefügt hat. ${ }^{5}$ Die $\S \S 298$ ff. StGB stellen wettbewerbsbeschränkende Absprachen bei Ausschreibungen ${ }^{6}$ und Bestechlichkeit und Bestechung im geschäftlichen Verkehr unter Kriminalstrafe. ${ }^{7}$

\section{Legitimation der Straftaten gegen den Wettbewerb?}

Die Frage nach Sinn und Zweck, also die Legitimation, dieser Straftatbestände soll im Folgenden erörtert werden. Besteht die Notwendigkeit, den freien Wettbewerb durch Normen des Strafgesetzbuches zu schützen, oder sollte ein entsprechender Schutz nicht besser über die Vorschriften des Kartell-, Ordnungswidrigkeiten- oder Wettbewerbs-

* Der Beitrag basiert auf der Monographie des Verfassers „Strafrechtlicher Schutz des Wettbewerbs? Eine kritische Analyse von Sinn und Zweck der Straftatbestände zum Schutz des Wettbewerbs" Frankfurter kriminalwissenschaftliche Studien, Band 120, Peter LangVerlag, Frankfurt am Main 2009.

1 Otto, ZRP 1996, S. 300.

2 Vgl. Diemer/Krick in: Münchener Kommentar, StGB, 2006, § 299 Rn 34.

3 Lüderssen, BB 1996 (Beilage), S. 1, 2.

4 Gegen die Fehlinterpretation von Strafrecht als Kampfinstrument und die illiberale Funktionslogik eines „Bekämpfungsrechts“ wendet sich zu Recht Sander, Grenzen instrumenteller Vernunft im Strafrecht, Eine Kritik der Präventionsdoktrin aus strafrechtsgeschichtlicher und empirischer Perspektive, 2007, S. 307 ff.

5 Gesetz vom 13.8.1997 (BGBl. I, S. 2038).

6 Zum Tatbestand des § 298 StGB und seiner Entstehung eingehend Grützner, Die Sanktionierung von Submissionsabsprachen - Eine Untersuchung der bestehenden Möglichkeiten einer Bekämpfung von Submissionsabsprachen unter besonderer Berücksichtigung des § $298 \mathrm{StGB}$, 2002.

7 Zur Abgrenzung der Straftatbestände untereinander: BGHSt 49, 201 ff. $=$ BGH JZ 2005, S. $47 \mathrm{ff}$. 
rechts erfolgen? Dabei gilt es insbesondere zu hinterfragen, ob der Wettbewerb als solcher überhaupt strafrechtlichen Schutz genießen kann, und ob die strafrechtlichen Steuerungsmöglichkeiten innerhalb der marktwirtschaftlichen Ordnung - besonders aus rechtsstaatlicher Sicht - ein taugliches Instrument für den Schutz von Rechtsgütern der Volkswirtschaft darstellen. ${ }^{8}$ Die Implementierung von Straftatbeständen ist nämlich nur dann legitim, wenn hierdurch menschlich und gesellschaftlich schützenswerte Interessen, die als strafrechtliche Rechtsgüter qualifiziert werden können, geschützt werden. ${ }^{9}$

\section{Fehlende Strafwürdigkeit der Straftaten gegen den Wettbewerb}

$\mathrm{Ob}$ und welche Angriffe gegen schützenswerte Interessen einen Straftatbestand erfordern, beurteilt sich anhand der Kriterien Strafwürdigkeit und Strafbedürftigkeit. ${ }^{10}$ Den Straftatbeständen des 26. Abschnitts fehlt es allerdings bereits an der erforderlichen Strafwürdigkeit, soweit sie den freien Wettbewerb als primäres Rechtsgut schützen sollen. Strafwürdig ist ein Verhalten, wenn es ein Maß an Sozialgefährlichkeit oder Sozialschädlichkeit erreicht, das geeignet ist, die Beziehungen innerhalb der Gesellschaft erheblich zu gefährden oder zu schädigen. ${ }^{11}$ Die Beurteilung ist anhand zweier Kriterien vorzunehmen. Sie erfolgt im Hinblick auf den Wert, der dem angegriffenen Interesse in der Gesellschaft beigemessen wird und der Verwerflichkeit der Angriffshandlung, also Erfolgs- und Handlungsunwert. ${ }^{12}$ Im Hinblick auf den Wettbewerb als vorrangig zu schützendes Interesse fehlt es den Straftaten gegen den Wettbewerb an dem notwendigen Erfolgsunwert, denn der freie Wettbewerb ist entgegen der herrschenden Meinung ${ }^{13}$ kein strafrechtlich zu schützendes Rechtsgut.

Dass der freie Wettbewerb ein strafrechtlich zu schützendes Gut sein muss, um die Aufnahme der Wettbewerbsdelikte ${ }^{14}$ in das Strafgesetzbuch zu legitimieren, folgt aus der Aufgabe des Strafrechts, Rechtsgüter und die Freiheit des Einzelnen zu schützen. ${ }^{15}$

Allerdings lassen sich weder der Rechtsgutslehre, der Verfassung noch dem einfachen Recht überzeugende Argumente entnehmen, die einen strafrechtlichen Schutz des Wettbewerbs rechtfertigen könnten. So ist es bisher - soweit ersichtlich - nicht gelungen,

8 Vgl. Albrecht, Kriminologie, Eine Grundlegung zum Strafrecht, 3. Aufl., 2005, S. 292 ff.

9 Vgl. Hassemer, GS-Schlüchter, 2002, S. 133, 152.

10 Frisch, FS-Stree/Wessels, 1993, S. 69; Otto, NJW 1979, S. 681, 683; Fürhoff, AG 2003, S. 80,81 .

11 Zum Begriff der Strafwürdigkeit: Otto, Jura 1989, S. 24, 27; Otto, NJW 1979, S. 681, 683; Fürhoff, AG 2003, S. 80, 85.

12 Vgl. Otto, NJW 1979, S. 681, 683 m.w.N; Günther, JuS 1978, S. 8, 13.

13 Statt vieler: Heine in: Schönke/Schröder, StGB, 27. Aufl., 2006, Vorbem. §§ $298 \mathrm{ff}$. Rn 2, $\S 298$ Rn 1, § 299 Rn 2; Tröndle/Fischer, StGB, 56. Aufl., 2006, § 298 Rn 2, § 299 Rn 2.

14 Der Begriff Wettbewerbsdelikte bezeichnet an dieser Stelle und im Folgenden die Straftatbestände $\S 298$ und $\S 299$ StGB.

15 Vgl. Naucke, Strafrecht, Eine Einführung, 10. Aufl., 2002, § 6 Rn 59 ff.; Hassemer, ZRP 1997, S. 316, 318. Zum Freiheitsschutz als Aufgabe eines rechtsstaatlichen Strafrechts siehe Albrecht, Kriminologie, a.a.O., S. 93 ff.; Albrecht, Die vergessene Freiheit, Strafrechtsprinzipien in der europäischen Sicherheitsdebatte, 2003, S. 48 f. 
eine abschließende Anzahl von Rechtsgütern zu bestimmen. ${ }^{16}$ Insofern kann anhand der unterschiedlichen Rechtsgutskonzeptionen, einschließlich der personalen Rechtsgutslehre, keine abschließende Aussage getroffen werden, ob der freie Wettbewerber ein strafrechtlich zu schützendes Rechtsgut ist.

Zwar wird vereinzelt davon ausgegangen, ${ }^{17}$ die Wertordnung der Verfassung biete eine verlässliche Richtschnur für die Entscheidung der Frage, welche Rechtsgüter so hochrangig sind, dass sie strafrechtlichen Schutz verdienen. ${ }^{18}$ Ein Blick in die Verfassung ist jedoch ebenfalls wenig hilfreich, denn die Verfassung kann nur im Hinblick auf zu schützende Individualrechtsgüter als Erkenntnisquelle herangezogen werden. Demgegenüber lassen sich ihr keine konkreten Kriterien oder Handlungsanweisungen entnehmen, um festzustellen, welche überindividuellen Rechtsgüter so bedeutend sind, dass sie strafrechtlichen Schutz verdienen sollten. ${ }^{19}$ Der freie Wettbewerb gilt als überindividuelles Rechtsgut. ${ }^{20} \mathrm{Er}$ ist weder grundrechtlich abgesichert noch wird er an anderer Stelle im Grundgesetz ausdrücklich gewährleistet. Das Grundgesetz enthält - im Gegensatz zur Weimarer Reichsverfassung (Art. 151 ff. WRV) - keine ausdrücklichen Regelungen über die Ordnung des Wirtschaftslebens. ${ }^{21}$ Es statuiert weder eine bestimmte Wirtschaftsverfassung noch verlangt es eine bestimmte Wirtschaftspolitik. ${ }^{22}$ Vielmehr ist die Verfassung, wie das Bundesverfassungsgericht mehrfach betont hat, wirtschaftspolitisch neutral und überlässt die Wirtschaftspolitik grundsätzlich dem gesetzgeberischen Ermessen. ${ }^{23}$ Enthält die Verfassung für den Gesetzgeber weder verbindliche Vorgaben für die konkrete Ausgestaltung des Wirtschaftssystems noch die Gewährleistung eines bestimmten Wirtschaftssystems, kann ihr auch kein Pönalisierungsgebot im Hinblick auf den Schutz einer konkreten, den gesetzlichen vorgegebenen Rahmen ausfüllenden Ausgestaltung eines Wirtschaftssystems entnommen werden. ${ }^{24}$ Insofern kann das Grundgesetz nicht herangezogen werden, um den strafrechtlichen Schutz des freien Wettbewerbs zu legitimieren.

16 Lüderssen, BB 1996, Beilage 11, S. 6.; Hassemer/Neumann in: Kindhäuser/Neumann/Paeffgen, StGB, 2. Aufl., 2005, Vor $§ 1$ Rn 144 sprechen sich sogar dafür aus, den Begriff des Rechtsguts offen zuhalten, um nicht von vornherein den Blick auf relevante Phänomene zu verengen.

17 Baumann/Weber/Mitsch, Strafrecht, Allgemeiner Teil, 11. Aufl., 2003, § 3 Rn 12; zu diesem Verweis kritisch: Appel, Verfassung und Strafe, Zu den verfassungsrechtlichen Grenzen staatlichen Strafens, 1998, S. 354 f., 372 ff.; kritisch zum Rekurs auf die objektive Wertordnung im Strafrecht auch Braum, KritV 1995, S. 371, 375 ff.

18 So auch Sax, Grundsätze der Strafrechtspflege, in: Bettermann/Nipperdey/Scheuner, Die Grundrechte, Handbuch der Theorie und Praxis der Grundrechte, Band III/2, 1959, S. 909, 911 ff.; Otto, NJW 1979, S. 681, 683 m.w.N.

19 So auch Stächelin, Strafgesetzgebung im Verfassungsstaat, 1998, S. 89; vgl. auch Lenckner/ Eisele in: Schönke/Schröder, a.a.O., Vorbem. §§ 13 ff. Rn 10; Eser, FS-Mestmäcker, 1996, S. $1005,1019$.

20 BT-Drs. 13/5584, S. 12.

21 Rittner/Dreher, Europäisches und deutsches Wirtschaftsrecht, 2008, § 2 Rn 47.

22 Badura, JuS 1976, S. 205, 208 f.; BVerfGE 50, 290, 337.

23 BVerfGE 4, 7, 17 f.; BVerfGE 50, 290, 336 ff.; Pieroth/Schlink, Grundrechte, Staatsrecht II, 15. Aufl., 1999, § 21 Rn 814; Badura, JuS 1976, S. 205, 208; kritisch Herzog in: Maunz/Dürig, GG, Loseblattsammlung, Stand: Juni 2007, Art. 20, II. Abschnitt VIII. Rn 60.

24 So auch Grützner, a.a.O., S. 443. 
Wenn jedoch weder anhand der Rechtsgutslehre noch anhand der verfassungsrechtlichen Werteordnung eindeutig festgestellt werden kann, ob der Wettbewerb ein strafrechtlich zu schützendes Rechtsgut darstellt, ist anhand von Struktur und Funktion des freien Wettbewerbs zu ermitteln, ob er als strafrechtlich zu schützendes Gut anzuerkennen ist. Dies ist nicht der Fall. Der Wettbewerb ist für sich genommen keine Institution, die in den Rang eines strafrechtlich geschützten Rechtsguts gehoben werden kann. Ihm fehlen die hierfür erforderlichen Konturen. ${ }^{25}$ Diese werden vielmehr von wirtschaftlichen und politischen Opportunitäten bestimmt. Insofern sind unlautere Verhaltensweisen im wirtschaftlichen Wettbewerb nicht als extrem sozialschädliche Angriffe auf ein überragendes Gemeinschaftsgut, sondern allenfalls als Zuwiderhandlungen gegen staatliche Lenkungsmaßnahmen zu verstehen.

Überdies ist und kann es nicht Aufgabe des Strafrechts sein, einzelne, die Wirtschaftsordnung mitkonstituierende Institutionen, wie den Wettbewerb, um ihrer selbst Willen als überindividuelles Rechtsgut zu schützen. ${ }^{26}$ Vielmehr hat sich eine zweckrationale und liberale Kriminalpolitik auch im Wirtschaftsstrafrecht am Schutz rechtlich garantierter Bedingungen individueller Freiheitssphären, also am Schutz von Vermögen und Dispositionsfreiheit des Einzelnen zu orientieren. Zwar erfüllt der freie Wettbewerb in unserer Gesellschaft freiheitssichernde und machtbegrenzende Funktionen, also besondere Funktionen, die dem gemeinschaftlichen Zusammenleben sowie dem Schutz und der Entfaltung des Einzelnen dienen. ${ }^{27}$ Jedoch handelt es sich bei dem Wettbewerb um ein normatives Gebilde, zusammengesetzt aus den Normen der staatlichen Wirtschaftsordnung und -lenkung. Ein einzelner Verstoß gegen diese Normen kann den Wettbewerb in seinem Bestehen niemals - auch nicht abstrakt - gefährden. ${ }^{28}$

Im Gegensatz zu anderen Rechtsgütern gibt es beim Wettbewerb keine feste oder zumindest klar eingrenzbare Institution oder Größe. Da die freie Marktwirtschaft und der Wettbewerb Abläufe und Prozesse im Wirtschaftsleben darstellen, die ständigen Veränderungen unterworfen sind, müssen wettbewerbsbeeinträchtigende Handlungen stets anhand der unterschiedlichen und wechselnden Verhältnisse des Wirtschaftslebens beurteilt werden. Das bedeutet, wer Verstöße gegen die Freiheit des Wettbewerbs tatsächlich feststellen und normativ festhalten will, muss Abwägungsprozesse vornehmen, die von den unvorhersehbaren Wechseln des Wirtschaftslebens und der Wirtschaftspolitik bestimmt sind. Solche Abwägungsprozesse sind aber mit einem Regelsystem, das, wie das Strafrecht, dem Legalitätsprinzip und dem Bestimmtheitsgebot ${ }^{29}$ unterliegt, nicht zu vereinbaren. ${ }^{30}$ Der freie Wettbewerb als Ergebnis wirtschaftspolitischer und

25 Vgl. König, JR 1997, S. 397, 402; vgl. auch die Kritik bei Hassemer, NStZ 1989, S. 553, 557, dass insbesondere Universalrechtsgüter im Bereich der Wirtschaft besonders vage und großflächig formuliert sind.

26 Hohmann in: Münchener Kommentar, a.a.O., § 298 Rn 3; vgl. Grützner, a.a.O., S. 461.

27 Vgl. Heine in: Schönke/Schröder, a.a.O., Vorbem. §§ 298 Rn 2; Wiedemann, Handbuch des Kartellrechts, 1999, § 1 Rn 2; vgl. Rittner/Dreher, a.a.O., § 15 Rn 44.

28 Vgl. Kuhlen, FS-Lampe, 2003, S. 743, 748; so auch Bottke in: Bausteine des europäischen Wirtschaftsstrafrechts, Madrid-Symposium für Klaus Tiedemann, 1994 S. 109, 111 f.; a.A. wohl Pragal, ZIS 2006, S. 63, 70.

29 Zur Bedeutung von Legalitätsprinzip und Bestimmtheitsgebot in einem freiheitlich-demokratischen Staat siehe Albrecht, Die vergessene Freiheit, a.a.O., S. 47 ff., S. 79 ff.; Albrecht, Kriminologie, a.a.O., S. 104 ff., S. 115 ff.

30 Vgl. Lüderssen, StV 1997, S. 318, 320. 
wirtschaftsrechtlicher Abläufe stellt daher kein Rechtsgut dar, das strafrechtlichen Schutz genießen sollte. ${ }^{31}$

\section{Mangelnde Strafbedürftigkeit der Straftaten gegen den Wettbewerb}

Überdies fehlt den kriminalisierten Verhaltensweisen der Wettbewerbsdelikte die erforderliche Strafbedürftigkeit. Hierunter versteht man, dass die Strafe unerlässliches Mittel sein muss, um die Gesellschaft vor strafwürdigen Rechtsgutsbedrohungen oder -verletzungen zu schützen und die Rechtsordnung zu bewahren. ${ }^{32}$ Dieses Zweckmoment in der Strafe steht der Kriminalisierung eines Verhaltens dann entgegen, wenn andere, weniger gravierende Eingriffe als die Pönalisierung des Verhaltens einen besseren oder zumindest den gleichen Erfolg versprechen, zur Verfügung stehen. ${ }^{33}$ Das Strafrecht darf nicht lückenlos jedes moralisch vorwerfbare Verhalten oder gar die Gesamtheit sozialer und gesellschaftlicher Verflechtungen erfassen, sondern soll lediglich einzelne, vom Gesetzgeber als besonders sozialschädlich erachtete Verhaltensweisen unter Strafe stellen. Das Strafrecht hat also fragmentarischen Charakter, d.h. es ist lückenhaft und lässt Freiräume. ${ }^{34}$ Der gesetzgeberische Einsatz von Strafrecht darf wegen des verfassungsrechtlichen Verhältnismäßigkeitsprinzips immer nur ultima ratio sein. ${ }^{35}$ Das bedeutet, das Strafrecht darf nur dann eingesetzt werden, wenn andere Mittel der sozialen Problemlösung und des Rechtsgüterschutzes, wie etwa zivilrechtliche Klagen, polizei- oder ordnungsrechtliche Anordnungen oder nichtstrafrechtliche Sanktionen, versagen. ${ }^{36}$

Diesen rechtsstaatlichen und freiheitssichernden Anforderungen werden die Wettbewerbsdelikte im 26. Abschnitt des Strafgesetzbuchs in keiner Weise gerecht, denn die Aufnahme der Wettbewerbsdelikte ist nicht als ultima ratio anzusehen. So stehen andere, mildere Regelungsmaterien zur Verfügung, um wettbewerbswidrige Verhaltensweisen effizient zu ahnden. Die kartellrechtlichen Bußgeldtatbestände, der Straftatbestand des Betrugs und zivilrechtliche Reaktionsmöglichkeiten im Gesetz gegen den unlauteren Wettbewerb sind geeignete, aber zugleich mildere Regelungen, um wettbewerbswidrige Verhaltensweisen zu sanktionieren. Daneben können Präventivmaßnahmen, wie beispielsweise die Einrichtung von Compliance-Funktionen ${ }^{37}$ in Wirtschaftsunternehmen, unlautere Handlungen im Geschäftsverkehr und damit im Wettbewerb wirksam verhindern. Ein Rückgriff auf das Strafrecht ist daher nicht erforderlich.

31 So auch Rittner/Dreher, a.a.O., § 23 Rn 132, nach deren zutreffender Auffassung der Wettbewerb oder typische Wettbewerbsbeschränkungen nicht zu einem Straftatbestand taugen, da es sich beim Wettbewerb um ein offenes, nicht determiniertes System handelt.

32 Möschel, Zur Problematik einer Kriminalisierung von Submissionsabsprachen, 1980, S 43 f.; Kasper, Die Erheblichkeitsschwelle im Bereich des Umweltstrafrechts, insbesondere bei $\S 324$ : Bagatellkriminalität im Umweltstrafrecht, 1997, S. $24 \mathrm{f}$.

33 Otto, Jura 1989, S. 24, 27; vgl. Frisch, FS-Stree/Wessels, 1993, S. 69, 77 ff.

34 Statt vieler: Naucke, a.a.O., § 2 Rn 13; vgl. Volk, JZ 1982, S. 85, 88.

35 Vgl. Fürhoff, AG 2003, S. 80, 82; Kartte/Portatius, BB 1975, S. 1169, 1170; vgl. BVerfGE 39, 1, 47; BVerfGE 88, 203, 258.

36 Kempf, NJW 1997, S. 1729, 1730.

37 Der Begriff Compliance wird verwendet, um die Einhaltung von Gesetzen und Richtlinien, aber auch freiwilligen Kodizes in Unternehmen zu bezeichnen. 
Die kriminalpolitische Legitimität der Wettbewerbsdelikte ist auch deshalb zu verneinen, weil diese Straftatbestände als abstrakte Gefährdungsdelikte ausgestaltet sind $^{38}$ und auf den Eintritt eines konkreten Schadens verzichten. ${ }^{39}$ Insbesondere an der Einführung des $\S 298$ StGB, dem die gesetzgeberische Intention zugrunde lag, die Schwierigkeit, einen Vermögensschaden i.S.d. § 263 StGB nachzuweisen, zu beseitigen, zeigt sich, dass die moderne Strafgesetzgebung mit dem Ziel, Institutionen wie den Wettbewerb zu schützen, sich von rechtstaatlichen Traditionen entfernt. Zu diesen Traditionen zählt unter anderem, dass nicht Gefährdungsdelikte, sondern Erfolgs- und Verletzungsdelikte die Normalform von Straftatbeständen darstellen sollten. ${ }^{40}$ Denn mit der Deliktsform der abstrakten Gefährdungsdelikte, die auf einen konkreten Schaden als Tatbestandsmerkmal verzichtet, ist eine beträchtliche Vorverlagerung der Strafbarkeit und damit eine rechtsstaatlich bedenkliche Ausweitung des Strafrechts verbunden. ${ }^{41}$

\section{Keine nennenswerte Effizienz in der Strafverfolgungspraxis}

Schließlich spielen die Straftaten gegen den Wettbewerb in der Praxis der Strafverfolgung keine bedeutende Rolle. ${ }^{42}$ So sind die den amtlichen Statistiken entnommenen Fallzahlen sehr niedrig. Nur wenige Fälle der Wettbewerbsdelikte werden der Polizei überhaupt bekannt und noch weniger von der Justiz abgeurteilt. Daher lässt sich anhand der Statistiken allenfalls die Aussage treffen, dass diese kriminalisierten Verhaltensweisen tatsächlich vorkommen. Eine Angabe der Häufigkeit ist aufgrund begrenzter Aussagekraft und Unbestimmtheit des Dunkelfeldes nur sehr eingeschränkt möglich. Auch im Vergleich zu den übrigen Straftaten fällt der Anteil der Wettbewerbsdelikte sehr gering aus. Welchen tatsächlichen Schaden Volks- und Marktwirtschaft durch die Wettbewerbsdelikte erleiden, kann letztlich nur geschätzt werden. Immaterielle Schäden können zwar angenommen, aber ebenfalls nicht eindeutig belegt werden. Diese praktische Bedeutungslosigkeit lässt sich als bedeutendes Indiz dafür sehen, dass es dem Gesetzgeber nicht gelungen ist, sachlich angemessenen Strafrechtsschutz gegen die von

38 So die überwiegende Meinung: Fischer, a.a.O., § 298 Rn 3 a, § 299 Rn 2 b; Heine in: Schönke/ Schröder, a.a.O., § 298 Rn 2 § 299 Rn 2; Diemer/Krick in: Münchener Kommentar, a.a.O., $\S 299$ Rn 2; Klusmann in: Wiedemann, Handbuch des Kartellrechts, a.a.O., § 56 Rn 13; a.A.: Grützner, a.a.O., S. 489 ff.; Hohmann in: Münchener Kommentar, a.a.O., § 298 Rn 6 ff.; Pasewaldt, ZIS 2008, S. 84, 85 sieht in $§ 298$ StGB ein konkretes Gefährdungsdelikt; Krey/ Hellmann, Strafrecht Besonderer Teil, Band 2, Vermögensdelikte, 13. Aufl., 2002, § 15 Rn 534 c sehen ebenso wie Walter, GA 2001, S. 131 ff. in $§ 298$ ein Verletzungsdelikt.

39 Kempf/Schilling, wistra 2007, S. 41, 47; kritisch zu der Ausgestaltung abstrakter Gefährdungsdelikte Hassemer, ZRP 1992, S. 378, 381; Hassemer, NStZ 1989, S. 553, 558.

40 Hassemer, ZRP 1992, S. 378, 379.

41 Vgl. Volk, JZ 1982, S. 85, 87; Naucke, KritV 1993, S. 135, 145; Nach Albrecht, KritV 1993, S. 163, 169 war bereits im Zusammenhang mit dem ersten und zweiten Wirtschaftskriminalitätsgesetz zu beobachten, dass zahlreiche Normen des ersten und zweiten Wirtschaftskriminalitätsgesetzes eine Vorverlagerung des Strafrechtschutzes mit dem erklärten Ziel des Gesetzgebers darstellen, prozessualen Beweisschwierigkeiten hinsichtlich der Voraussetzungen des zentralen strafwürdigen Betrugstatbestandes zu entgehen.

42 So auch Fischer, a.a.O., Vor § 298 Rn 4; Dannecker in: Kindhäuser/Neumann/Paeffgen, a.a.O., Vor $\S 298$ Rn 6. 
ihm als vermeintlich sozialschädlich eingestuften Verhaltensweisen im Wettbewerb zu bieten. $^{43}$

\section{Freiheitlich-liberales Strafrecht versus Effizienzsteigerung der Strafverfolgung}

Vor diesem Hintergrund besteht keine Notwendigkeit, den freien Wettbewerb durch die Straftatbestände im Strafgesetzbuch zu schützen. Die Wettbewerbsdelikte zeigen exemplarisch, dass die neueren wirtschaftlichen Straftatbestände nicht mehr ausschließlich auf den Schutz von Individualrechtsgütern abzielen. Vielmehr geht es um den Schutz von Funktionen, Institutionen oder Allgemeininteressen. Für diese Art von Rechtsgütern lassen sich nicht immer individualisierbare Schädigungen angeben, sondern es geht um diffuse Schadensbegriffe wie Funktionsstörungen oder wie bei den $\S \S 298 \mathrm{ff}$. StGB um die Beeinträchtigung des freien Wettbewerbs. Um eine möglichst effektive „Bekämpfung“ von wettbewerbsbeeinträchtigenden Verhaltensweisen zu erzielen, wird sowohl bei $\S 298$ StGB als auch $\S 299$ StGB auf den Nachweis eines konkreten Vermögensschadens verzichtet. Der Anwendungsbereich des Strafrechts wird damit weit im Vorfeld einer Verletzung von Individualrechtsgütern eröffnet. Als Folge hiervon stehen die Wettbewerbsdelikte im Widerspruch zu einem freiheitlich-liberalen Strafrecht, denn sie werden weder dem strafrechtsbegrenzenden Charakter des Subsidiaritätsprinzips noch dem fragmentarischen Charakter des Strafrechts gerecht. Außerstrafrechtliche Regelungsmaterien und gesellschaftlich-marktwirtschaftliche Selbstregulierungskräfte stehen zur Verfügung, bleiben aber aufgrund einer vermeintlichen Effizienzsteigerung der strafrechtlichen „Bekämpfung“ wettbewerbsbeeinträchtigender Verhaltensweisen unberücksichtigt.

Es ist verfehlt, aus der Gefährlichkeit korruptiver Entwicklungen den Schluss zu ziehen, diese müssten mit neuen Straftatbeständen, Erhöhung der Strafdrohungen oder Beseitigung von Strafbarkeitsvoraussetzungen ,bekämpft“ werden, denn es gibt auch andere Möglichkeiten: Nicht das Strafrecht, sondern das Wirtschaftsrecht, das Verwaltungsrecht, oder die Betriebswirtschaftslehre eröffnen Kontroll- und Steuerungsmöglichkeiten, ${ }^{44}$ damit das Strafrecht die ihm im Rechtsstaat zukommende Rolle spielen, damit es ultima ratio von wirtschaftlichen Problemlösungen sein kann und nicht zur prima oder gar zur sola ratio wird. ${ }^{45}$

Naucke hat die „Reduzierung eines ausgeuferten Strafrechts als das große strafrechtliche Problem der Zeit" bezeichnet. "46 Leben, Unverletzlichkeit und Freiheit der Person sowie Eigentum bleiben die Rechtsgüter, die Strafrecht - auf absehbare Zeit - zu sichern und nach Verletzungen immer wieder herzustellen hat. Das ist der Kernbereich von Strafrecht, ohne den auch eine moderne Gesellschaft nicht auskommt. Der Schutz des Wettbewerbs zählt nicht hierzu und sollte anderen Regelungsmaterien vorbehalten bleiben.

43 Vgl. Otto, Jura 1989, S. 24, 27.

44 Albrecht, Die vergessene Freiheit, a.a.O., S. 169.

45 Vgl. Hassemer, ZRP 1997, S. 316, 321.

46 Naucke, Die Grenzen des Strafrechts. Eine Problemskizze in 7 Thesen, Archivum Iuridicum Cracoviense, Bd. 27/28, 1994-1995, S. 65 ff. 